\title{
Carta de sensibilidade ambiental ao óleo: origem, evolução e tendências
}

\author{
Environmental sensitivity map for oil spill: origin, evolution and trends
}

\author{
Patrick Thomaz de Aquino Martins ${ }^{1,2}$, Paulina Setti Riedel², João Carlos Carvalho Milanelli² \\ ${ }^{1}$ Universidade Estadual de Goiás, Minaçu - GO, Brasil \\ ${ }^{2}$ Universidade Estadual Paulista “Júlio de Mesquita Filho", Rio Claro - SP, Brasil
}

\begin{abstract}
Resumo
O presente trabalho tem como objetivo explanar a carta de sensibilidade ambiental ao derramamento de óleo (Carta SAO) por meio de uma revisão de literatura. Normatizada a partir da década de 1970, a Carta SAO constitui elemento norteador ao planejamento de ações de contingência em episódios de derramamento de óleo. São abordados a sua gênese, as perspectivas atuais, a situação do cenário brasileiro, culminando com uma rápida abordagem do principal ferramental utilizado na elaboração das Cartas SAO. Há uma ênfase nos modelos baseados no sistema de Índice de Sensibilidade Ambiental (ISA) da National Oceanic and Atmospheric Administration (NOAA), por ser este o mais adotado no Brasil e no mundo. Há destaque às Cartas SAO produzidas aos ambientes marinho e costeiro, reflexo do histórico de aplicação. A oportunidade de evolução metodológica está no desenvolvimento de propostas ao mapeamento da sensibilidade ao óleo dos ambientes terrestres, uma vez que estes são desprovidos de um sistema mundialmente aceito.
\end{abstract}

Palavras-chave: Carta SAO, petróleo, revisão, derramamento.

\begin{abstract}
This paper aims is explain the environmental sensitivity map to oil spill, by the literature review. Standardized from the 1970s, the environmental sensitivity map to oil spill is an element to guide the planning of contigency actions in oil spill episodes. Are discussed its Genesis, the current prospects, the situation of the Brazilian scene, culminating in a rapid approach of the main tools used for this specific mapping. There is an emphasis on models based on the Environmental Sensitivity Index (ESI) from National Oceanic and Atmospheric Administration (NOAA), because this is the most widely adopted in Brazil and worldwide. There are highlight to maps produced to the marine and coastal environments, reflection of application history. The methodological evolution opportunity is to develop proposed to terrestrial environmental sensitivity mapping to oil, once they are devoid of a ESI accepted worldwide.
\end{abstract}

Keywords: Sensitivity map, oil, review, spill. 


\section{Introdução}

Apesar do domínio de avançadas técnicas de transporte de petróleo e seus derivados nos modais atualmente utilizados (dutoviário, ferroviário, marítimo e rodoviário), as condições adversas (humanas, ambientais e de materiais) enfrentadas por esse sistema induzem à ocorrência de acidentes.

Nestes casos, planos de emergência são necessários para que o potencial poluidor do episódio possa ser minimizado e controlado no menor espaço de tempo possível. A Carta de Sensibilidade Ambiental ao Óleo (Carta SAO) é uma ferramenta que auxilia decisões desta competência.

A Carta SAO é um documento cartográfico legalmente obrigatório no planejamento de contingência, na avaliação geral de danos e na implantação de ações de resposta aos incidentes de poluição por petróleo e derivados (BARBOSA et al., 2010).

O conhecimento da origem, evolução e tendências desse documento abre novas perspectivas à sua elaboração, bem como no desenvolvimento de metodologias e na aplicação em nichos geográficos específicos ou ambientes poucos explorados. Nesse sentido, o presente trabalho tem como objetivo revisar e discutir os aspectos técnicos básicos da carta de sensibilidade ambiental ao derramamento de óleo, desde a sua gênese a perspectivas futuras.

\section{Origem e pressupostos}

O mapeamento da sensibilidade ambiental vem sendo utilizado desde a década de 1970 e representa uma importante ferramenta técnico-gerencial à priorização dos ambientes a serem protegidos, e também dos esforços e recursos a serem aplicados ou concentrados, em episódios acidentais e emergências, no intuito de reduzir as conseqüências ambientais tanto do derrame quanto dos procedimentos de limpeza (ARAUJO et al, 2002).

O método de classificação e mapeamento da sensibilidade ambiental especificamente voltado a derramamentos de óleo foi desenvolvido por um grupo de pesquisadores da Divisão de Pesquisas Costeiras da Universidade da Carolina do Sul (UCS) durante o estudo de três grandes derramamentos de óleo ocorridos nos navios petroleiros "Metula", "Urquiola" e “Jakob Maersk" (MICHEL et al., 1978). Duas publicações, complementares, procedentes desse grupo foram as que lançaram as bases aos estudos de sensibilidade ambiental ao óleo como é realizada hoje, que são Gundlach \& Hayes (1978) e Michel et al. (1978).

Gundlach \& Hayes (1978) apresentaram e aplicaram o "Índice de Vulnerabilidade ao Derrame de Óleo" a partir da classificação dos ambientes cos- teiros em uma escala (índices) de 1 a 10 em termos de vulnerabilidade potencial ao dano por derramamento de óleo. Esta classificação foi baseada na interação da linha de costa com os processos físicos que controlam a deposição do óleo, persistência ou longevidade do óleo no ambiente e extensão do dano biológico.

De maneira similar, o esquema de classificação de índices de Michel et al. (1978) foi atrelado principalmente ao modo de ocorrência e à longevidade do óleo em diferentes ambientes costeiros, embora considerando também a suscetibilidade biológica e facilidade de limpeza manual.

Chama atenção em ambos os estudos pioneiros o uso do termo vulnerabilidade (vulnerability) em detrimento da palavra sensibilidade (sensitivity), tratadas como sinônimo nessas publicações, mesmo sendo a segunda, de acordo com Romero (2009), conceitualmente complementar a primeira. Complementação esta dada a partir da compreensão de outro termo, a suscetibilidade.

Esta posição é confirmada por Zacharias e Gregr (2005), os quais entendem a sensibilidade como o grau no qual o ambiente responde ao stress que, neste caso, é o óleo A suscetibilidade é a probabilidade ou tendência de um ambiente ser atingido pelo óleo. Por fim, a vulnerabilidade, por sua vez, é a resultante da totalização entre a sensibilidade e a probabilidade (suscetibilidade) do ambiente ser atingido em episódios de derramamento de óleo (ROMERO, 2009).

Outro aspecto que deve ser ressaltado é o fato de que as Cartas SAO inicialmente se resumiam ao mapeamento dos índices de sensibilidade/vulnerabilidade ao óleo, sem a associação com elementos socioeconômicos ou faunísticos.

Quase que concomitante à proposta de Carta SAO do grupo da USC, uma derivação de mapa de sensibilidade ao óleo foi sugerida por Walker et al. (1978). Esta proposta, entretanto, foi concebida para ambientes terrestres e se baseia em experimentos com óleo cru e óleo diesel, realizados em comunidades de plantas da Baía de Prudhoe, Alaska, Estados Unidos.

Nas três décadas que transcorreram desde os primeiros estudos publicados de mapeamento da sensibilidade ambiental ao óleo até o presente, muitos métodos foram propostos, visando tanto a classificação dos ambientes/índices quanto o desenvolvimento do ferramental para sua implementação. Nesses, há métodos que incorporam dados socioeconômicos, e outros que dão destaque a aspectos biológicos (ARAUJO et al., 2002). Outros ainda dão maior liberdade à interpretação do mapa, apresentando um inventário de recursos, sem referenciar áreas prioritárias à proteção e/ou limpeza (ARAUJO et al., 2002).

Os anos de 1990 podem ser considerados como a década em que todo o mundo concentrou esforços 
e difundiu o uso das cartas de sensibilidade a óleo como ferramenta técnico-gerencial em caso de derramamento de petróleo e derivados, tendo como marco o emblemático acidente do Exxon Valdez ocorrido em 1989 (LEACOCK, 2005).

Há, entre os métodos de elaboração e mapeamento de cartas de sensibilidade, os que requerem mais tempo e um grande número de informações quando comparados aos convencionais. Embora esses métodos "sofisticados" forneçam mapas com informações valiosas, é difícil a sua reprodução, devido à dificuldade de levantamento das informações. Uma consequência diante desta variedade de métodos foi a dificuldade operacional na comparação ou avaliação integrada de áreas diferentes com sistemas de mapeamento distintos (ARAUJO et al, 2002).

O Guia da ARPEL, Associação Regional das Companhias de Petróleo e Gás Natural na América Latina e no Caribe (WOTHERSPOON, 1997), exemplifica a diversidade de métodos em Cartas SAO citando estudos que utilizam cálculo matricial com base em sensibilidade ambiental; impactos culturais; impactos econômicos; impactos arqueológicos; tipos de costa; aplicabilidade de contramedidas de resposta, segundo as correntes marítimas; dentre outras.

A diversidade de métodos e classificações particulares a diferentes áreas de estudo ou de cunho técnico-científico específico, mesmo contribuindo à discussão e resultando no avanço metodológico, acabava por restringir a comparação entre áreas distintas, tornando difícil a sua utilização (PINCINATO, 2007).

Visando instituir um sistema padronizado de cartas de sensibilidade ao óleo para aplicar nos Estados Unidos, em 1993 a National Oceanic and Atmospheric Administration (NOAA) estabeleceu um guia para a produção destas Cartas (MICHEL \& DAHLIN, 1993). Este guia vem sendo constantemente atualizado (HALLS et al., 1997; PETERSEN et al., 2002) sendo amplamente utilizado em diversas partes do mundo.

O mapeamento das Cartas de sensibilidade ao óleo amplamente utilizado atualmente é composto por três principais componentes: um sistema de ranqueamento de linha de costa em escala de 1 a 10, no qual quanto maior o índice, maior a sensibilidade; identificação dos recursos biologicamente sensíveis ao óleo; e o uso humano dos recursos de valor comercial, recreacional ou de subsistência (JENSEN et al.1998).

As Cartas de sensibilidade ao óleo frequentemente têm seu emprego adaptado à realidade dos ambientes locais, agregando pequenas variações de método e representação simbólica em relação ao proposto pelo NOAA (Figura 1). Apesar dessas nuances, o básico do mapeamento tem permanecido constate na maioria dos projetos, suportando o desenho conceitual original (GUNDLACH et al., 2001)

Dentre os países onde a carta de sensibilidade ao óleo e suas variações têm sido aplicadas destacamse: Austrália (McENALLY \& THOMPSON, 1984), Egito (WENNINK \& NELSON-SMITH, 1979; HANNA, 1995; LOTFY, 2004), Emirados Árabes (JENSEN et al., 1993), Espanha (CASTANEDO et al., 2009), Estados Unidos (GUNDLACH \& HAYES, 1978; MICHEL et al. 1978), Índia (KANKARA \& SUBRANIAN, 2007), Israel (ADLER \& INBAR, 2007), Noruega (WESLAWSKI et al., 1997; MOE et al., 2000), Reino Unido (KRISHNAN, 1995), Trinidad e Tobago (NANSINGH \& JURAWAN, 1999), Venezuela (GARCIA, 1986), dentre outros.

No Brasil, as Cartas de sensibilidade também têm sido empregadas desde a década de 80 . Devido à heterogeneidade ambiental presente no território nacional, estas passaram por um processo de padronização visando ordenar a sua confecção e otimizando seu uso pelos diversos atores envolvidos.

\section{A Carta de sensibilidade ao óleo no Brasil}

Seguindo a tendência internacional, no Brasil os trabalhos de mapeamento da sensibilidade ambiental ao óleo se iniciaram focando os ambientes costeiros e marinhos. E foi neste contexto que elas mais evoluíram.

As cartas de sensibilidade nacionais também ganharam notoriedade após o acidente envolvendo o Exxon Valdez. Um pouco antes, porém, Awazu e Poffo (1986) propuseram áreas a serem protegidas quando da ocorrência de derrames de petróleo e derivados no Litoral Norte de São Paulo. Um ano após, na mesma região, Maldonado et al. (1987) realizaram o mapeamento da "vulnerabilidade dos ambientes costeiros do município de Ubatuba ao impacto de derramamentos de óleo".

Estes esforços já demonstravam a preocupação na proteção dos ambientes costeiros e a efetividade do mapeamento como ferramenta em casos de derramamento de óleo nestes ambientes.

Ainda que em 1993 a Petrobras já divulgasse o primeiro mapa de sensibilidade produzido pela instituição, apenas a partir de 1996 as Cartas de sensibilidade ao óleo produzidas pela empresa foram padronizadas nos moldes da metodologia NOAA (ARAUJO et al., 2006). O “Manual básico para elaboração de mapas de sensibilidade ambiental a derrames de óleo no sistema Petrobras: ambientes costeiros e estuarinos", produzido pela Petrobras (ARAUJO et al., 2002), foi o primeiro guia produzido no Brasil.

O Manual da Petrobras foi utilizado como 

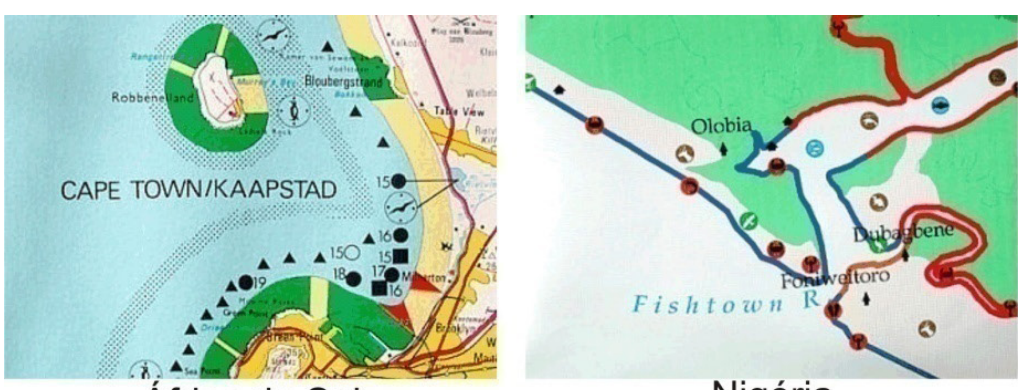

Nigéria
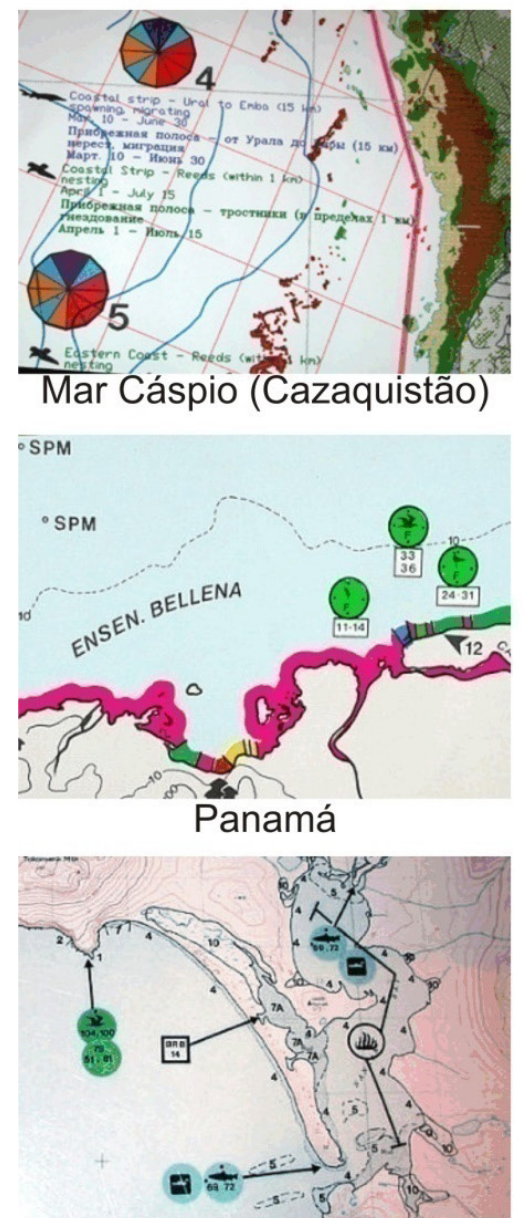

Alasca (EUA)

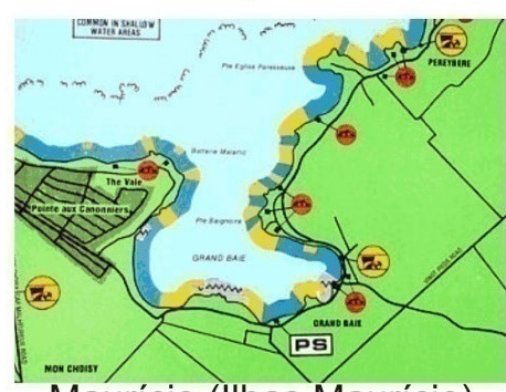

Maurício (Ilhas Maurício)

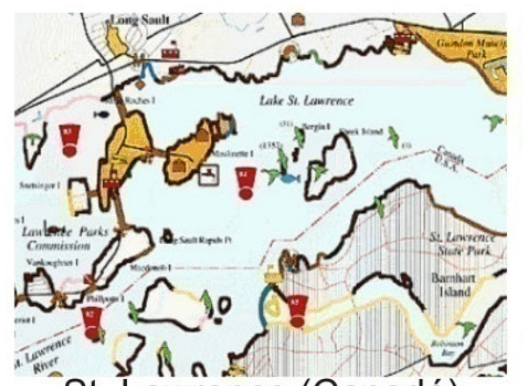

St. Lawrence (Canadá)

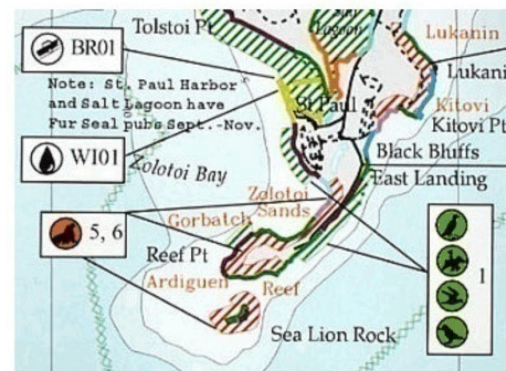

Alasca (EUA)

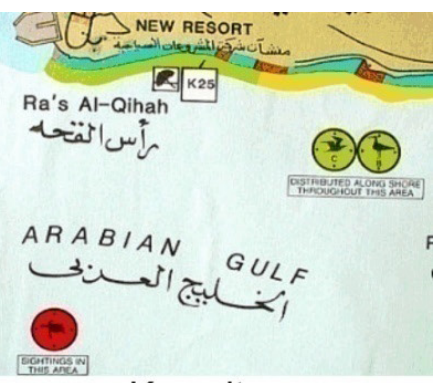

Kuwait

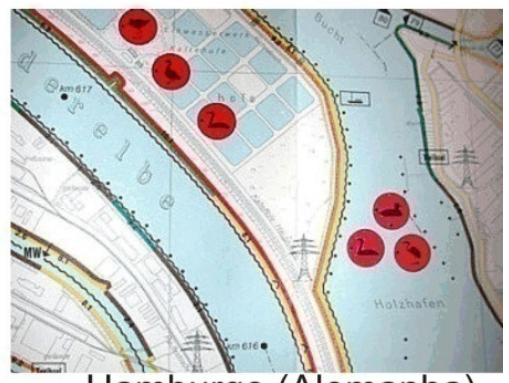

Hamburgo (Alemanha)
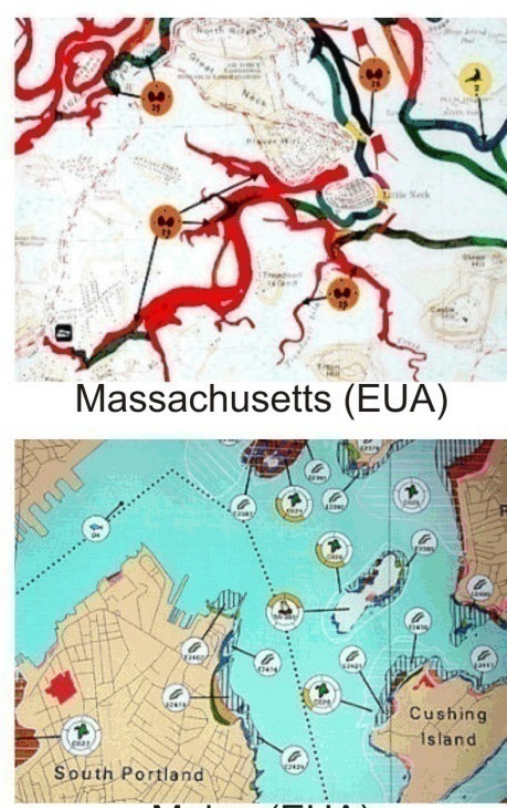

Maine (EUA)

Figura 1. Exemplos de representação de Cartas de sensibilidade a óleo em diferentes lugares do mundo. Fonte: E-Tech International Inc (2010).

subsídio à elaboração das "Especificações e normas técnicas para a elaboração de cartas de sensibilidade ambiental para derramamentos de óleo" (MMA, 2002; MMA, 2004; ARAUJO et al., 2006), documento referência ao mapeamento dos índices de sensibilidade ambiental ao óleo no país. Foi neste documento que foi instituída a ferramenta CARTAS SAO (Cartas de sensibilidade ao óleo) no Brasil.

Apesar da substancial contribuição do Manual da Petrobras para a composição das Especificações do MMA, o método das Cartas de sensibilidade se baseou também amplamente no guia da NOAA.
De modo geral, os estudos compreendendo as Cartas SAO no Brasil, apesar de recentes e concentrados na zona marítima e costeira, estão em franco desenvolvimento e expansão, realizados na maioria das bacias marítimas onde há extração de petróleo.

Apesar da produção técnico-cientifica estar dispersa em diferentes contextos, certamente este arsenal de informação contribui para o amadurecimento da ferramenta. Dentre as contribuições, podem ser citados trabalhos de conclusão de cursos acadêmicos, como Monografias (ARAUJO, 2005; LACERDA, 2006; MONTANARI, 2006; MULER, 2008; NASCIMENTO, 


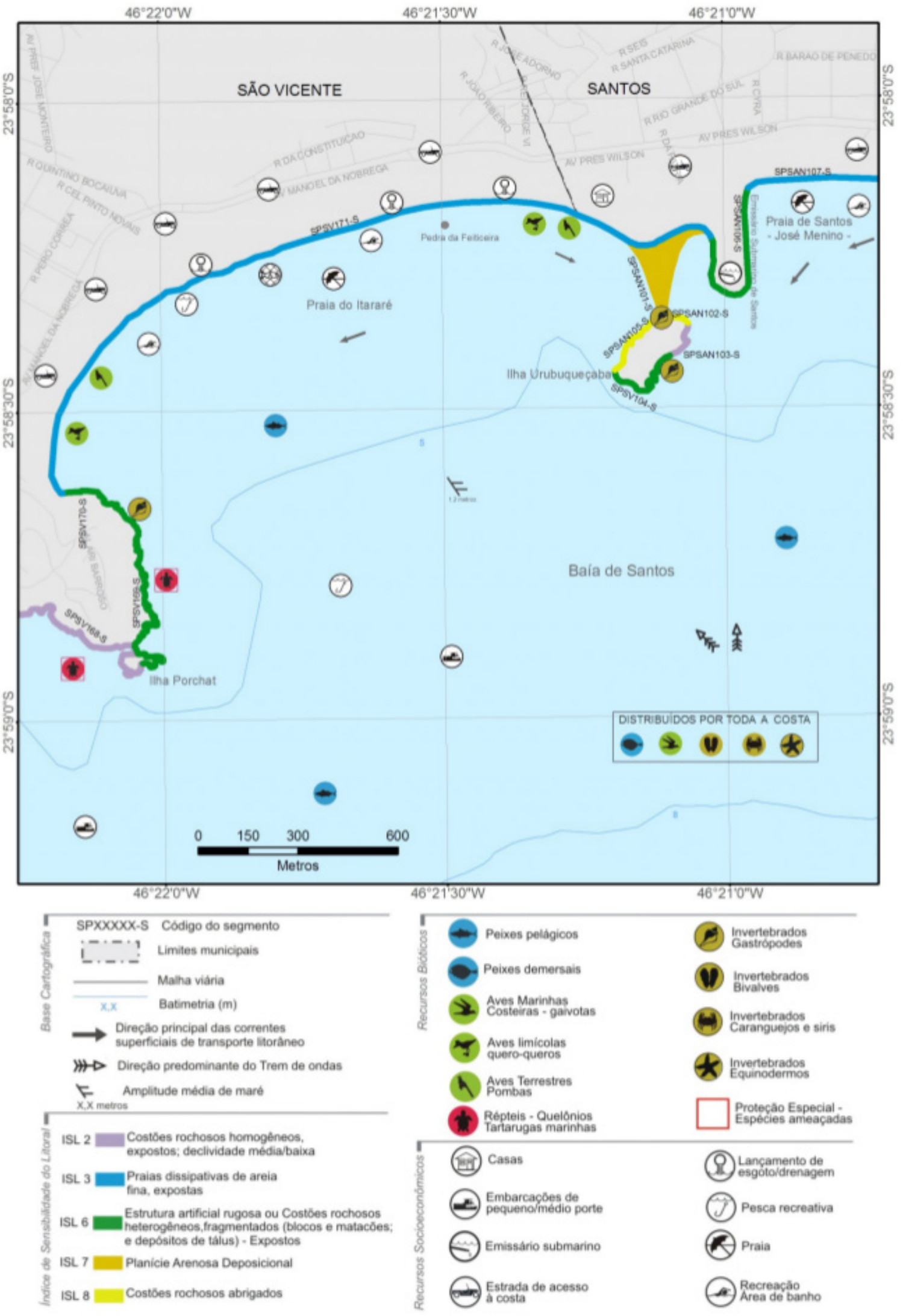

Figura 2. Exemplo de Carta SAO (Norte da linha de costa da Baía de Santos). Fonte: Perinotto et al. (2010). 
2008; SILVA, 2006; TINÓS, 2007), Dissertações (CUNHA, 2009; LIMA, 2007; LOTFY, 2004; PERINOTTO, 2010; PINCINATO, 2007; ROCHA, 2009; WIECZOREK, 2006) e Teses (ALMEIDA, 2008; ROMERO, 2009); trabalhos em anais (ALCÂNTARA; SANTOS, 2005; ALMEIDA, 2009; BOULHOSA; MENDES, 2009; CABRAL et al., 2007; CARVALHO; GHERARDI, 2003, 2005; CASTRO et al., 2003, 2005; GHERARDI et al., 2001; GONÇALVES; SOUZA FILHO, 2005; NOVAES, 2007); artigos (ARAUJO et al., 2007; BELLOTTO; SAROLLI, 2008; CANTAGALLO et al., 2008; GHERARDI et al., 2008; LIMA et al., 2008; NOERNBERG et al., 2008; ROCHA -OLIVEIRA et al., 2008; Silva et al., 2008); livros/atlas (ARAUJO et al, 2006; FREIRE, 2004; GHERARDI \& CABRAL, 2007); além de consultorias, cujo produtos finais (cartas, relatórios, etc.) são de caráter confidencial.

Segundo as Especificações do MMA (2004), as cartas SAO incluem três tipos de informações principais: (1) sensibilidade dos ecossistemas (costeiros e marinhos), de acordo com as características geomorfológicas dos habitats e persistência natural do óleo e sua limpeza, classificando os ambientes em um ranking que vai de 1 a 10, de forma que quanto maior o índice, maior a sensibilidade; (2) recursos biológicos, com informações do meio biótico, detalhando inventário de espécies presentes, e sensíveis ao óleo; e (3) recursos/atividades socioeconômicas existentes e suscetíveis aos derramamentos de óleo ou às ações de resposta e combate (Figura 2).

Três também são as escalas de elaboração das Cartas SAO: (1) estratégica, de abrangência regional/ marítima; (2) tática, de escala intermediária/todo o litoral da bacia; e (3) operacional / de detalhe, locais de alto risco/sensibilidade. Estes níveis são baseados no Plano Cartográfico para o Mapeamento de Sensibilidade Ambiental ao Óleo, o qual adotou as Bacias Marítimas como unidades cartográficas (MMA, 2004).

Vale ressaltar que os níveis de elaboração supracitados estão diretamente correlacionados com o volume derramado, ou seja, quanto maior o volume, menor a escala utilizada, sendo que, no caso de grandes descargas, pode haver a necessidade de mapeamento em diversas escalas. As diferentes escalas são complementares e integradas, sendo utilizadas em níveis de gestão distintos da emergência.

Atualmente, os estudos de cartas SAO no Brasil têm buscado a adaptação dos Índices de Sensibilidade Ambiental - ISA aos ambientes continentais. Essa adaptação está intrinsecamente ligada ao adensamento de trabalhos no litoral e no mar, concomitantemente à flagrante e emergente necessidade destas ferramentas como instrumento de gestão de emergências com vazamentos de óleo fluviais e terrestres. Certamente a experiência adquirida ao longo de mais de 20 anos no Brasil facilita extremamente a replicação e adaptação da metodologia para estes ambientes.

\section{Outros mapas de sensibilidade ambiental ao óleo}

Assim como aconteceu nos ambientes costeiros e marinhos, o mapeamento da sensibilidade ambiental em outros ambientes também resultou na diversidade metodológica, embora bem menor que o primeiro. Neste caso, há alguns mapas que buscam se alinhar com o guia NOAA, através do seu sistema de índices e simbologia. Outros, porém, se contrapõem a tal proposta utilizando métodos alternativos.

Fora da zona costeira e marinha, há um volume crescente de estudos que propõem utilizar o mapeamento da sensibilidade ambiental ao óleo para outros ambientes. Naturalmente, o cenário que apresentou maiores contribuições foram os dulciaquícolas, com destaque aos ambientes fluviais.

A NOAA, seguindo esta tendência, incluiu na atualização dos índices do seu guia a sensibilidade de ambientes lacustres, fluviais e palustres (NOAA, 1995; PETERSEN et al., 2002). Embora este sistema seja bem aceito, carece de discussão quanto à integração e equivalência dos índices envolvendo ambientes distintos.

Com essa inclusão, outros estudos passaram a aplicar ou propor métodos semelhantes, e estes ambientes já contam com uma gama de estudos relevantes, e.g. Hayes et al. (1995); Hayes et al. (1997); Zengel et al. (2001a); Zengel et al. (2001b); Araujo et al. (2006); Ferreira \& Beaumord (2008), dentre outros.

Ainda no meio dulciaquícola, há tentativas de se aplicar métodos de Carta SAO em Bacias Hidrográficas (WVI, do inglês, Watershed Vulnerability Index) (JENSEN et al., 1998; WEATHERS et al., 2009), o que seria uma extensão dos índices aplicados aos rios em uma unidade de gestão baseada no meio físico continental e em escala regional.

Nos ambientes terrestres, as áreas adjacentes aos modais de transporte e às bases de refino ou armazenamento são as que permitem a aplicação mais efetiva de índices de sensibilidade ambiental ao óleo. Nesse contexto, já existem iniciativas metodológicas para o mapeamento da sensibilidade ao óleo, como o trabalho de Walker et al., em 1978, propondo três classes de sensibilidades aos ambientes: (1) baixa, (2) moderada e (3) alta.

Contemporaneamente, os trabalhos de Mendes et al. (2005) e Gundlach et al. (2005), se destacam por resgatar a abordagem da sensibilidade para áreas de dutos. Em ambos os casos, há a busca de alinhamento ao método NOAA, utilizando variações de classificação e símbolos daquele órgão, o que é um aspecto positivo visando à integração das ferramentas. 
A composição de Carta de sensibilidade a óleo para rodovias e ferrovias ainda está em estágio embrionário, com relativamente poucas publicações representativas.

A sensibilidade ambiental ao óleo em áreas rodoviárias tem nos trabalhos de Mattos (2008) e de Martins (2012) dois dos poucos estudos pioneiros nesse tipo de modal. A principal diferença metodológica envolvendo estas propostas está na opção pela classificação em três níveis de sensibilidade ambiental ao óleo (Alta, Moderada e Baixa) do primeiro trabalho, ao passo que o segundo utiliza como base as classes e simbologias da NOAA.

No modal ferroviário, Silva (2010) se destaca pelo pioneirismo ao propor uma metodologia para o mapeamento da vulnerabilidade ambiental no transporte ferroviário de produtos perigosos, perfeitamente aplicável em caso de derramamentos de óleo.

Ainda no meio terrestre, Santos (2008) inova ao elaborar uma carta de sensibilidade ambiental com três níveis de sensibilidade, aplicada a postos de combustíveis.

\section{As geotecnologias e as Cartas SAO}

Os principais problemas com as Cartas SAO no início de sua concepção e aplicação eram a dificuldade e custo de sua atualização. De acordo com Sorensen (1995), na produção de um atlas típico composto por Cartas SAO, por exemplo, uma equipe obtinha a informação de várias bases de dados, sintetizava o material e transcrevia diretamente no mapa base. Quando era necessária uma atualização, o que ocorria em um curto espaço de tempo, todo esse procedimento deveria ser refeito.

Até 1989, os mapas de sensibilidade ambiental eram produzidos em papel, com distribuição limitada, justamente devido aos custos de produção e sem meios para uma rápida atualização (PETERSEN et al. 2002). A partir desse ano, o uso das geotecnologias passou a modernizar o sistema de produção desses mapas, tornando-os mais dinâmicos e acessíveis.

As principais vantagens do uso das geotecnologias na produção das Cartas SAO são: a identificação e extração de informações dos ecossistemas a partir da análise visual e/ou digital de imagens de sensoriamento remoto; o armazenamento e organização dos dados em um Banco de Dados Geográficos (BDG), permitindo a rápida identificação, recuperação e atualização dos dados; e a integração das imagens com dados coletados in situ para a geração de mapas e simulações de cenários através de análise espacial (GHERARDI \& CABRAL, 2007).
A metodologia para a criação de Cartas SAO utilizando a tecnologia SIG, de acordo com Jensen et al. (1998), envolve: reunião com especialistas em recursos locais e regionais; documentação da localização de recursos biológicos, de uso antrópico e ambiental; compilação de informação em mapas e tabelas; digitalização de dados espaciais e informação de atributo em formato digital específico; desempenho da classificação da sensibilidade ambiental; produção inicial de mapas Índice de Sensibilidade Ambiental e tabelas de atributos à revisão; incorporação/edição de especialistas em recursos locais; produção final das Cartas SAO e tabulação dos produtos; e liberação dos produtos digitais (inclusive os metadados) para disseminação.

Existem atualmente dezenas de sistemas sensores e programas institucionais voltados ao sensoriamento remoto com as características mais variáveis (resolução: espectral, temporal, espacial e radiométrico; plataforma: aéreo, sub-orbital e orbital; fonte de energia: ativo ou passivo; região do espectro atuante: óptica - termais e de energia solar refletida, ou de microondas; forma de capação: imageadores e não-imageadores; polarização: horizontal e vertical; ângulo de inclinação; etc.)

A variedade de possibilidades de instrumentos sensores, interpretação e processamento das imagens, e dos métodos e aplicativos utilizados ao processamento é refletida de diversas formas na produção das Cartas SAO. Deste modo, as propostas, estudos e aplicações de Cartas SAO dificilmente utilizam um método rígido, não só no uso do sensoriamento remoto, mas em todas as geotecnologias.

\section{Considerações finais}

A consolidação de um sistema de mapeamento da sensibilidade ambiental a partir daquele adotado pela NOAA e utilizado como base por uma gama de órgãos ambientais, nos quais se incluem a ARPEL e o MMA, foi um avanço fundamental às ações de combate em eventos de derramamento de óleo.

É importante para o continuo amadurecimento e melhoria da estratégia a diversidade de propostas que surgem a cada ano, uma vez que aumenta a discussão em torno das técnicas de mapeamento de sensibilidade.

A integração dos conceitos de sensibilidade e suscetibilidade nos mapas de vulnerabilidade foi um avanço relevante na gestão ambiental dos vazamentos de óleo.

É claro o desbalanceamento entre os mapeamentos de sensibilidade ambiental marinhos/costeiros e os dos ambientes interiores (fluviais e terrestres). 
Diante da intensa demanda de crescimento da indústria do petróleo (exploração, produção, transporte e armazenamento) e do uso dos modais de transporte terrestre (dutovias, ferrovias e rodovias) é fundamental que sejam concentrados esforços para o desenvolvimento e implantação oficial destas técnicas no Brasil.

É urgente, ainda, a necessidade sistematização de índices, aceitos globalmente, aplicados à sensibilidade ao óleo de ambientes terrestres, uma vez que o ranqueamento mais bem aceito atualmente não abarca tais espaços e não é passível de aplicação integral.

O intenso crescimento observado na indústria do petróleo no Brasil, resultante da nova era Pré-Sal, demanda também que sejam alavancadas as técnicas e metodologias de mapeamento ambiental e de gestão integrada, a ela vinculadas.

A crescente participação dos diversos segmentos da sociedade (governo, academia, iniciativa privada e ONGs,) neste cenário indica que o país possui corpo técnico competente e capaz de superar o desafio de mapear as suas áreas de influência e regiões relevantes do ponto de vista ambiental e sócio econômico, garantindo a implantação de ferramentas eficientes para a gestão de crise envolvendo vazamentos de petróleo e derivados no novo cenário que se instala no Brasil.

\section{Agradecimentos}

À Agência Nacional do Petróleo, Gás Natural e Biocombustíveis, ANP; à Petróleo Brasileiro S.A., PETROBRAS; e ao Programa para a Formação de Recursos Humanos para o Setor de Petróleo e Gás, PRH-05 (Convênio UNESP/ANP/MCT/FINEP), pela concessão de bolsa de doutorado ao primeiro autor. Aos pareceristas avaliadores, pelas valorosas contribuições.

\section{Referências}

ADLER, E., M. INBAR. Shoreline sensitivity to oil spills, the Mediterranean coast of Israel: Assessment and analysis. Ocean \& Coastal Management, v. 50, n.1-2, p. 24-34. 2007.

ALCÂNTARA, E. H.; SANTOS, M. C. F. V. Mapeamento de Áreas de Sensibilidade Ambiental ao Derrame de Óleo na Região Portuária do Itaquí, São Luís, MA-Brasil. In: Simpósio Brasileiro de Sensoriamento Remoto, 12. (SBSR), 2005, Goiânia. Anais... 2005. p. 3605-3617.

ALMEIDA, E. F. Sensibilidade ambiental a derramamentos de óleo dos ecossistemas costeiros da Região do Cabo Norte, Amapá: a contribuição do sensoriamento remoto. 2008. Tese (Doutorado em Engenharia Civil) - Universidade Federal do Rio de Janeiro, Rio de Janeiro, 2008. 202p.

ALMEIDA, E. F. Uso de Dados Multisensor para geração de Cartas de Sensibilidade Ambiental a Derramamentos de Óleo, nos Ecossistemas Costeiros da Região do Cabo Norte, Amapá. In: Simpósio Brasileiro de Sensoriamento Remoto, 14. (SBSR), 2009, Natal. Anais... 2009. p. 4543-4551.

ARAUJO, S. I; SILVA, G. H.; MUEHE, D. C. E. H. Manual básico para elaboração de mapas de sensibilidade ambiental a derrames de óleo no sistema Petrobras: ambientes costeiros e estuarinos. Rio de Janeiro: 2002. 134p.

ARAUJO, R. S. Determinação do Índice de Sensibilidade do Litoral (ISL) ao derramamento de óleo (ISL) para as regiões Norte e Centro-Norte do Estado de Santa Catarina (SC). 2005. Monografia (Graduação em Oceanografia) - Universidade do Vale do Itajaí, Itajaí, 2005. 190p.

ARAUJO, S. I; SILVA, G. H.; MUEHE, D. CARVALHO, M. T. M; MENEZES, P. M. L.; ALCÂNTARA, A. V.; VARGAS, M. A. M.; TAKAHASHI, L. T. Mapas de sensibilidade ambiental a derrames de óleo: ambientes costeiros, estuarinos e fluviais. Rio de Janeiro: Petrobras: Jauá, 2006. 168p.

ARAUJO, R. S.; PETERMANN, R. M.; KLEIN, A. H. F.; MENEZES, J. T.; SPERB, R. M.; GHERARDI, D. F. M. Determinação do Índice de Sensibilidade do Litoral (ISL) ao Derramamento de Óleo para as Regiões Norte e Centro-Norte da Costa de Santa Catarina (SC). Gravel, v. 5, n. 1, p. 47-73, 2007.

AWAZU, L. A.; POFFO, I. R. F. Mapeamento de áreas a serem protegidas quando da ocorrência de derrames de petróleo e derivados no Litoral Norte de São Paulo. CETESB: São Paulo, 1986. 40p.

BARBOSA, C. C. A.; VASCONCELOS, T. L.; VALDEVINO, D. S.; SÁ, L. A.C. M. Estruturação de base cartográfica para mapeamento de sensibilidade ambiental a derrames de óleo ao longo do litoral pernambucano. In: III Simpósio Brasileiro de Ciências Geodésicas e Tecnologias da Geoinformação, 2010, Recife/PE. Anais... Recife/ PE, 2010. P. 001-008. 
BELLOTTO, V. R.; SAROLLI, V. M. M.

Mapeamento da sensibilidade ambiental ao derramamento de óleo e ações de resposta para a região costeira e área portuária de Imbituba, SC, Brasil. Braz. J. Aquat. Sci. Technol., v. 12, n. 2, p. 115-125, 2008.

BOULHOSA, M. B. M.; MENDES, A. C. Mapeamento dos Índices de Sensibilidade Ambiental ao derramamento de óleo através de imagens SPOT 5, na região portuária de vila do conde - Barcarena - PA. In: Simpósio Brasileiro de Sensoriamento Remoto, 14. (SBSR), 2009, Natal. Anais... 2009. p. 3597-3603.

BUCKLEY, R. C. Environmental sensitivity mapping - what, why and how. Mineral and the Environment, v. 4, p 151. 1982.

CABRAL, A. P.; GHERARDI, D. F. M.; BAPTISTA, E.; MUEHE, D.; NICOLODI, J. L.; CARVALHO, L. R. Cartas de sensibilidade ambiental à poluição por óleo: bacias de Sergipe-Alagoas e Pernambuco-Paraíba. In: Simpósio Brasileiro de Sensoriamento Remoto, 13. (SBSR), 2007, Florianópolis. Anais... 2007. p. 3785-3788.

CANTAGALLO, C.; GARCIA, G. J.; MILANELLI, J. C. C. Mapeamento de sensibilidade ambiental a derramamentos de óleo do sistema estuarino de Santos, Estado de São Paulo. Braz. J. Aquat. Sci. Technol., v. 12, n. 2, p. 33-47, 2008.

CARVALHO, M.; GHERARDI, D. F. M. Uso de transformação IHS e classificação não supervisionada por regiões para o mapeamento da sensibilidade ambiental ao derramamento de óleo. In: Simpósio Brasileiro de Sensoriamento Remoto, 11. (SBSR), 2003, Belo Horizonte. Anais... 2003. p. 1515-1523.

CARVALHO, M.; GHERARDI, D. F. M. Modelagem de um banco de dados geográficos para o mapeamento da sensibilidade ambiental ao derramamento de óleo na zona costeira. In: Simpósio Brasileiro de Sensoriamento Remoto, 12. (SBSR), 2005, Goiânia. Anais... 2005. p. 2101-2108.

CASTANEDO, S.; JUANES, J. A.; MEDINA, R.; PUENTE, A.; FERNANDEZ, F.; OLABARRIETA, M.; POMBO, C. Oil spill vulnerability assessment integrating physical, biological and socio-economical aspects: Application to the Cantabrian coast (Bay of Biscay, Spain). Journal of Environmental Management, v. 91, n. 1, p. 149-159, 2009.
CASTRO, A. F.; AMARO, V. E.; VITAL, H. Desenvolvimento de um banco de dados geográficos em um ambiente SIG e sua aplicação na elaboração de mapas de sensibilidade ambiental ao derramamento de óleo em áreas costeiras do Estado do Rio Grande do Norte. In: Simpósio Brasileiro de Sensoriamento Remoto, 11. (SBSR), 2003, Belo Horizonte. Anais... 2003. p. 1533-1540.

CASTRO, A. F.; SOUZA, C. F.; AMARO, V. E.; VITAL, H. Automação de cartas de sensibilidade ambiental ao derramamento de óleo utilizando técnicas de geoprocessamento em áreas costeiras da porção setentrional do estado do Rio Grande do Norte. In: Simpósio Brasileiro de Sensoriamento Remoto, 12. (SBSR), 2005, Goiânia. Anais... 2005. p. 2109-2111.

CUNHA, F. P. Mapeamento de sensibilidade ambiental a derramamentos de óleo na região costeira de Bertioga - SP. 2009. Dissertação (Mestrado em Geociências e Meio Ambiente) Universidade Estadual Paulista Júlio de Mesquita Filho, Rio Claro, 2009. P?

E-TECH INTERNATIONAL INC. Environmental Sensitivity Mapping. Disponíve em: <http://www. oil-spill-info.com/Mainframes/Mainframe $\% 20$ Mapping.htm>. Acesso em: 04 fev. 2010.

FERREIRA, M. F.; BEAUMORD, A. C. Mapeamento da sensibilidade ambiental à derrames de óleo nos cursos de água da bacia do Rio Canhanduba, Itajaí, SC. Brazilian Journal of Aquatic Science and Technology. v. 12, n. 2, p. 61-72, 2008.

FREIRE, O. D. S. (Coord.). Atlas de sensibilidade ambiental ao óleo das macias marítimas do Ceará e Potiguar. Brasília: MMA, 2004. 56p.

GARCIA, N. Venezuela National Oil Spill Contingency Plan. In: Oil Spills Research in Tropical Environments and Contingency Planning in the Wider Caribbean. London: International Petroleum Industry Environmental Conservation Association. 1986.

GHERARDI, D. F. M.; CABRAL, A. P. (Coords.). Atlas de sensibilidade ambiental ao óleo da Bacia Marítima de Santos. Brasília: MMA, SMCQ, 2007. 116p. 
GHERARDI, D. F. M.; BRAGA, C. Z. F.; EICHENBERGER, C. Utilização de imagens TM Landsat para o mapeamento do índice de vulnerabilidade ambiental ao impacto por óleo da zona costeira entre Rio Grande do Norte e Ceará, Brasil. In: Simpósio Brasileiro de Sensoriamento Remoto, 10. (SBSR), 2001, Foz do Iguaçu. Anais... 2001. p. 595-597.

GHERARDI, D. F. M.; CABRAL, A. P.; KLEIN, A. H. F.; MUEHE, D. C. E. H.; NOERNBERG, M. A.; TESSLER, M. G.; SARTOR, S. M. Mapeamento da sensibilidade ambiental ao óleo da bacia marítima de Santos. Braz. J. Aquat. Sci. Technol., v. 12, n. 2, p. 11-31, 2008.

GONÇALVES, F. D.; SOUZA FILHO, P. W. M. Integração digital de imagens Radarsat-1 e Landsat-7 para o mapeamento dos índices de sensibilidade ambiental a derramamentos de óleo na Baía de Guajará (Belém-PA). In: Simpósio Brasileiro de Sensoriamento Remoto, 12. (SBSR), 2005, Goiânia. Anais... 2005. p. 1789-1796.

GUNDLACH, E. R.; HAYES, M. O. Vulnerability of coastal environments to oil spill impacts.

Marine Technology Society Journal. v. 12, p. 18-27. 1978.

GUNDLACH, E.; IMEVBORE, V. O; WITHERSPOON, B.; AINODION, J. Incorporating biodiversity into sensitivity maps of the Niger River delta. International Oil Spill Conference, $\mathrm{p}$. 391-403, 2001.

GUNDLACH, E. R.; CEKIRGE, M.; ANUL, C.; ORHAN, C.; SUTHERLAND, P. Pipeline and coastal environmental sensitivity mapping for the BTC pipeline system in Turkey. International Oil Spill Conference, p. 1-5, 2005.

HALLS, J.; MICHEL, J.; ZENGEL, S.; DAHLIN, J.; PETERSEN, J. Environmental Sensitivity Index Guidelines. National Oceanic and Atmospheric Administration. v. 2, 1997, 79p.

HANNA, R.G.M. An approach to evaluate the application of vulnerability index for oil spills in tropical Red Sea environments. Spill Science \& Technology Bulletin, v.2, n.2/3, p. 181-186, 1995.

HAYES, M. O.; MICHEL, J.; DAHLIN, J. A.; BARTON, K. Identifying and mapping sensitive resources for inland area planning. International Oil Spill Conference, p. 365-371, 1995.
HAYES, M. O.; MICHEL, J.; MONTELLO, T. M. The reach sensitivity index (RSI) for mapping river and streams. International Oil Spill Conference. p. 343-350. 1997.

JENSEN, J. R.; HALLS, J. N.; MICHEL, J. A System Approach to Environmental Sensitivity Index (ESI) Mapping for Oil Spill Contingency Planning and Response. Photogrammetric Engineering \& Remote Sensing. v. 64, n. 10, p. 1003-1014, 1998.

JENSEN, J.R.; NARUMALANI, S.; WEATHERBEE, O.; MURDAY, M.; SEXTON, W.J.; GREEN, C.J. Coastal environmental sensitivity mapping for oil spills in the United Arab Emirates using remote sensing and GIS technology. Geocarto International, v. 2, p. 5-13, 1993.

KANKARA, R. S.; SUBRAMANIAN, B. R.. Oil Spill Sensitivity Analysis and Risk Assessment for Gulf of Kachchh, India, using Integrated Modeling. Journal of Coastal Research. v. 23, n 5, p. 1251-1258, 2007.

KRISHNAN, P., 1995. Research report - A geographical information system for oil spills sensitivity mapping in the Shetland Islands. (United Kingdom). Ocean \& Coastal Management, v. 26, n. 3, p. 247-255, 1995.

LACERDA, C. S. Cartas de Sensibilidade Ambiental a Derramamentos de Óleo - Cartas SAO - para a Costa Oeste da Lagoa dos Patos, RS, Brasil. 2006. Monografia (Graduação em Oceanologia) - Fundação Universidade do Rio Grande, Rio Grande do Sul, 2006. p. 56.

LEACOCK, E. The Exxon Valdez oil spill. Environmental Disasters. New York: Facts On File, Inc. 2005 100p.

LIMA, M. V. Mapeamento de sensibilidade ambiental ao óleo do arquipélago de Ilha Bela SP. 2007. Dissertação (Mestrado em Geociências e Meio Ambiente) - Universidade Estadual Paulista Júlio de Mesquita Filho, Rio Claro, 2007. 2 vol.

LIMA, M. V.; DIAS-BRITO, D; MILANELLI, J. C. C. Mapeamento da sensibilidade ambiental a derrames de óleo em Ilhabela, São Paulo. Revista Brasileira de Cartografia, v. 60, n. 2, p. 145-154, 2008.

LOTFY, I. N. GIS-based Enviromental Sensitivity Index (ESI) mapping for oil spills: Case study in 
Sharm El-Sheikh, Egypt. 2004. (Dissertação de mestrado). Physical Land Resources, Universiteit Gent, Brussel, Bélgica, 2004104 p.

MALDONADO, S. R. C.; ISHIHATA, L.; POLETTE, M. Vulnerabilidade dos ambientes costeiros do município de Ubatuba ao impacto de derramamentos de óleo (Litoral Norte do Estado de São Paulo). São Paulo: CETESB. 1987. 57p.

MARTINELLI, M. Mapas de geografia e cartografia temática. São Paulo: Contexto, 3 ed., 2006. 112p.

MATTOS, M. B. C. Application of oil spill environmental sensitivity analyses to Brazilian road networks. International Oil Spill Conference, p. 169-175, 2008.

MENDES, R. F.; MINNITI, V.; LOPES, C. F.; MILANELI, J.; TORRES, C.; YOGUI, R.; RODRIGUES, G.; MARIZ, E. MARA Elaboração de metodologias para análise dos riscos ambientais. In: Rio Pipeline Conference \& Exposition, 2005, Rio de Janeiro. Anais... Rio de Janeiro, 2005.

MICHEL, J.; HAYES, M. O.; BROWN, P. J. Application of an oil spill vulnerability index to the shoreline of lower Cook Inlet, Alaska. Environment geology, v. 2, p. 107-117, 1978.

MICHEL, J.; DAHLIN, J. Guidelines for Development of Digital Environmental Sensitivity Index Atlases and Database. National Oceanic and Atmospheric Administration. 1993, 43p.

MMA. Ministério do Meio Ambiente.

Especificações e normas técnicas para a elaboração de cartas de sensibilidade ambiental para derramamentos de óleo. Rio de Janeiro: MMA, 2002, 22p.

MMA. Ministério do Meio Ambiente.

Especificações e normas técnicas para a elaboração de cartas de sensibilidade ambiental para derramamentos de óleo. Brasília: MMA, 2004, 107 p.

MOE, K.A.; SKEIE,G.M.; BRUDE, O.W.; LØVÅS, S.M.; NEDREBØ, M.; WESLAWSKI, J.M. The Svalbart intertidal zonne: a concept for the use of GIS in applied oil sensivity, vulnerability and impact analyses. Spill Science \& Technology Bulletin, v. 6, n. 2, p.187-206, 2000.
MONTANARI, T. Subsídios para um modelo socioeconômico de Pressão-Estado-Resposta (P/E/R) para sensibilidade litorânea ao derramamento de óleo. 2006. Monografia (Graduação em Oceanografia) - Universidade do Vale do Itajaí, Itajaí, 2006. 115p.

MULER, M. Implementação de um sistema de informação voltado à sensibilidade ambiental ao óleo do litoral sul paulista e avaliação das ações de resposta a derramamentos de óleo. 2008. Monografia (Graduação em Engenharia Ambiental) - Universidade Estadual Paulista Júlio de Mesquita Filho, Rio Claro, 2008. 68p.

NANSINGH, P.; JURAWAN, S. Environmental sensitivity of a tropical coastline (Trinidad, West Indies) to oil spills. Spill Science \& Technology Bulletin, v. 5, n. 2, p.161-172, 1999.

NASCIMENTO, P. H. R. Organização e implementação de um banco de dados para a sensibilidade ambiental ao óleo e avaliação de ações adequadas de resposta a derramamentos São Sebastião (SP). 2008. Monografia (Graduação em Engenharia Ambiental) - Universidade Estadual Paulista Júlio de Mesquita Filho, Rio Claro, 2008. 66p.

NOAA. National Oceanic and Atmospheric Administration. Sensitivity mapping of inland areas: Technical support to the Inland Area Planning Committee Working Group. USEPA Region 5. HAZMAT Report 95-4. Seattle: Hazardous Materials Response and Assessment Division, National Oceanic and Atmospheric Administration. 54 pp. 1995.

NOERNBERG, M. A.; ANGELOTTI, R.; CALDEIRA, G. A.; RIBEIRO DE SOUSA, A. F. Determinação da sensibilidade do litoral paranaense à contaminação por óleo. Braz. J. Aquat. Sci. Technol., v. 12, n. 2, p. 49-59, 2008.

NOVAES, R. C.; TAROUCO, J. E. F.; RANGEL, M. E. S.; DIAS, L. J. B. S. Análise da sensibilidade ambiental da parte ocidental da Ilha do Maranhão. In: Simpósio Brasileiro de Sensoriamento Remoto, 13. (SBSR), 2007, Florianópolis. Anais... 2007. p. 4089-4096.

NOVO, E. M. L. M. Sensoriamento remoto: princípios e aplicações. São Paulo: Blücher, 1992. 308p. 
PERINOTTO, R. R. C. Mapeamento de sensibilidade ao derrame de oleo dos ambientes costeiros dos municípios de São Vicente, Santos e Guarujá, SP. 2010 Dissertação (Mestrado em Geociências e Meio Ambiente) - Universidade Estadual Paulista Júlio de Mesquita Filho, Rio Claro, 2010. 2 vol.

PERINOTTO, R. R. C.; Riedel, P. S.; MILANELLI, J. C. C. Sensibilidade ambiental ao derrame de oleo da linha de costa da Baía de Santos, SP Brasil. Revista Brasileira de Cartografia. n. 63/4, p. 501-514, 2010.

PETERSEN, J.; MICHEL, J.; ZENGEL, S.; WHITE, M.; LORD, C.; PLANK, C. Environmental Sensitivity Index Guidelines. National Oceanic and Atmospheric Administration. v. 3.0. 2002. 89p.

PINCINATO, F. L. Mapeamento da sensibilidade ambiental a derramentos de óleo para a região costeira de São Sebastião e Caraguatatuba, litoral norte de São Paulo (SP), com uso de modelagem em SIG de sistema especialista baseado em conhecimento e árvore de decisão. 2007. Dissertação (Mestrado em Geociências e Meio Ambiente) - Universidade Estadual Paulista Júlio de Mesquita Filho, Rio Claro, 2007. 63p.

ROCHA, T C. F. R. Mapeamento da sensibilidade ambiental do litoral de Ubatuba-SP a vazamentos de petróleo. 2009. Dissertação (Mestrado em Geociências e Meio Ambiente) - Universidade Estadual Paulista Júlio de Mesquita Filho, Rio Claro, 2009.

ROCHA-OLIVEIRA, T. C.; KLEIN, A. H. F.; PETERMANN, R. M.; MENEZES, J. T.; SPERB, R. M. Determinação do Índice de Sensibilidade do Litoral (ISL) ao derramamento de óleo, para região Sudeste e Sul do Estado de Santa Catarina. Braz. J. Aquat. Sci. Technol., v. 12, n. 2, p. 91-114, 2008.

ROMERO, A. F. Mapa de vulnerabilidade ambiental ao óleo e cartas SAO. Trecho: Praia Grande - Ilha Comprida, Litoral Paulista. 2009. Tese (Doutorado em Geociências e Meio Ambiente) - Universidade Estadual Paulista Júlio de Mesquita Filho, Rio Claro, 2009.

ROSA, R. Geotecnologias na Geografia Aplicada. Revista do Departamento de Geografia (USP), São Paulo, v. 16, p 81-90. 2005.
SANTOS, S. M. Proposta de elaboração de carta de sensibilidade ambiental aplicada a postos de combustíveis em Rio Claro-SP. 2008. Monografia (Graduação em Engenharia Ambiental) Universidade Estadual Paulista Júlio de Mesquita Filho, Rio Claro, 2008. 50p.

SILVA, A. F. Determinação do Índice de Sensibilidade do Litoral (ISL) ao derramamento de óleo, na ilha de Santa Catarina e áreas de entorno. 2006. Monografia (Graduação em Oceanografia) Universidade do Vale do Itajaí, Itajaí, 2006. 98p.

SILVA, R. O. Mapeamento de Vulnerabilidade Ambiental para o Gerenciamento de Riscos no Transporte Ferroviário de Produtos Perigosos uma Proposta Metodológica. 2010. Dissertação (Mestrado em Tecnologia Ambiental) - Instituto de Pesquisas Tecnológicas do Estado de São Paulo, 2010. 245p.

SILVA, A. F.; KLEIN, A. H. F.; PETERMANN, R. M.; MENEZES, J. T.; SPERB, R. M.; GHERARDI, D. F. M. Índice de Sensibilidade do Litoral (ISL) ao derramamento de óleo, para a ilha de Santa Catarina e áreas do entorno. Braz. J. Aquat. Sci. Technol., v. 12, n. 2, p. 73-89, 2008.

SORENSEN, M. ARC/INFO marine spill GIS. Spill Science \& Technology Bulletin. v. 3, n. 1, p. 81-85, 1995.

TINÓS, T. M. Contribuição para a elaboração de cartas de sensibilidade ambiental para o derramamento de óleo: os impactos socioeconômicos na atividade turística em Ilhabela - SP. 2007. Monografia (Graduação em Geografia) - Universidade Estadual Paulista Júlio de Mesquita Filho, Rio Claro, 2007. 79p.

McENALLY, J. M.; THOMPSON, G. B. Coastal Resource Atlas for Oil Spills in Trial bay. Australian State Pollution Control Comission. 1984.

WALKER, D. A., WEBBER, P. J., EVERETT, K. R.; BROWN, J. Effects of crude and diesel oil spills on plant communities at Prudhoe Bay, Alaska, and the derivation of oil spill sensitivity maps. Arctic, v. 31, p. 242-256, 1978.

WEATHERS, D. H.; HAYES, M. O.; MICHEL, J. Reach Sensitivity Index Mapping of the Amite River Watershed in the Lake Pontchartrain Basin: A Tool for Watershed Restoration. Journal of Coastal Research. v. especial. P. 141-151, 2009. 
WENNINK, C.J.; NELSON-SMITH, A. Coastal Oil Pollution Evaluation Study for the Gulf of Suez and the Red Sea Coast of the Republic of Egypt. London: IMO. 1979.

WESLAWSKI, J. M.; WIKTOR, J.; ZAJACZKOWSKI, M.; FUTSAETER, G.; MOE, K. A. Vulnerability assessment of Svalbard intertidal zone for oil spills. Estuarine, Coastal and Shelf Science, v. 44, pp. 33-41. 1997.

WIECZOREK, A. Mapeamento de sensibilidade a derramamentos de petróleo do parque estadual da Ilha do Cardoso (PEIC) e áreas adjacentes. 2006.

Dissertação (Mestrado em Geociências e Meio Ambiente) - Universidade Estadual Paulista Júlio de Mesquita Filho, Rio Claro, 2006.

WOTHERSPOON, P.; MARKS, D.; SOLSBERG, L.; WEST, M. Guía para el desarrolo de mapas de sensibilidad ambiental para la planificación y respuesta ante derrames de hidrocarburos. Montevideo: ARPEL, 1997. 90p.

ZACHARIAS, M. A.; GREGR, E. J. Sensitivity and Vulnerability in Marine Environments: an Approach to Identifying Vulnerable Marine Áreas. Conservation Biology, v. 19, n.. 1, pp. 86-97. Fevereiro, 2005. DOI: 10.1111/j.15231739.2005.00148.x.

ZENGEL, S.; HAYES, M. O.; MICHEL, J.; WHITE, $\mathrm{M}$. Integrated planning front the mountains to the sea: environmental sensitivity mapping in the Caribbean. International Oil Spill Conference, p. 1-5, 2001a.

ZENGEL, S.; HAYES, M. O.; MICHEL, J.; ROBERTSON, A. Sensitive areas planning for inland South Florida: web and GIS tools. International Oil Spill Conference, p. 789-796, $2001 b$. 